\title{
Evolution of empiric vancomycin dosing in a neonatal population
}

\author{
Luiza Radu $^{1} \cdot$ Tanner Bengry $^{1} \cdot$ Albert Akierman $^{2} \cdot$ Belal Alshaikh $^{2} \cdot$ Kamran Yusuf $^{2} \cdot$ Deonne Dersch-Mills $^{1}$
}

Received: 24 April 2018 / Revised: 21 September 2018 / Accepted: 1 October 2018 / Published online: 19 October 2018

(c) Springer Nature America, Inc. 2018

\begin{abstract}
Background In 2014, we assessed the effectiveness of our neonatal vancomycin empirical dosing regimen (15-45 mg/kg/ day) which led to development of a revised regimen $(20-60 \mathrm{mg} / \mathrm{kg} / \mathrm{day})$.

Objective To validate the revised empirical vancomycin dosage regimen in achieving target troughs.

Methods The primary outcome of this multicenter retrospective before-and-after cohort study was the proportion of neonates in the present cohort achieving trough levels below, at or above target $(<10,10-20$ and $>20 \mathrm{mg} / \mathrm{L})$. Secondary outcomes included difference between cohorts (historical and present) in mean troughs and proportion of patients achieving target levels.

Results Out of 118 participants, 63 (53.39\%) achieved target troughs, 44 (37.29\%) had below target troughs and $11(9.32 \%)$ reached above target levels. Mean trough levels and proportion of patients achieving target levels were higher in the present versus historical cohort $(p<0.01$ for all comparisons).

Conclusions The revised empiric dosing regimen was more effective in achieving target serum trough concentrations.
\end{abstract}

\section{Introduction}

Although sepsis-related mortality rates have decreased over the past 30 years, sepsis is still a threat to neonatal survival, especially in low birth weight and preterm neonates [1]. Between 1989 and 2003, a downward trend in the number of early-onset sepsis cases and an increase in the number of late-onset sepsis (LOS) episodes has been observed. With recent infection prevention interventions, LOS rates have started to decrease; however, in comparison with early onset, LOS is still associated with higher occurrence rates and mortality [2].

A variety of organisms are responsible for LOS, with coagulase-negative staphylococcus (CoNS) being the most common organism involved [1, 3, 4]. Vancomycin is commonly used to treat CoNS infections [3-5]. Thus, vancomycin dose optimization is important to maximize therapeutic effectiveness while limiting toxicity and development of bacterial resistance [3]. This poses a therapeutic

Deonne Dersch-Mills

Deonne.Dersch-Mills@ahs.ca

Alberta Health Services, Pharmacy Services, Calgary, AB, Canada

2 Division of Neonatology, Department of Medicine, University of Calgary, Calgary, AB, Canada challenge, considering vancomycin's variable pharmacokinetics in neonates $[4,6]$ and limited evidence to support the variety of dosing recommendations in neonates [3].

Increased rates of methicillin-resistant Staphylococcus aureus (MRSA) eradication in adults have been observed with area under the $24 \mathrm{~h}$ concentration-time curve to minimum inhibitory concentration ratios $\left(\mathrm{AUC}_{24} / \mathrm{MIC}\right)$ greater than 400 , and thus this is often considered the ideal therapeutic target for all pathogens [7]. Targeting $\mathrm{AUC}_{24} / \mathrm{MIC}$ ratios is clinically unfeasible, and has not been validated in neonates $[3,5,8]$, and thus most centers utilize trough levels to assess vancomycin dosing $[4,5,8]$. In adults, trough concentrations of $10-20 \mathrm{mg} / \mathrm{mL}$ are recommended as a surrogate parameter for $\mathrm{AUC}_{24} / \mathrm{MIC} \geq 400$ when treating pathogens with an MIC $\leq 1 \mathrm{mg} / \mathrm{L}$ [7]. There is emerging pharmacokinetic data that suggest the correlation between $\mathrm{AUC}_{2} / \mathrm{MIC}$ values and trough concentrations differs in neonates, and lower trough levels may be acceptable [9], but, until further research, many clinicians continue to use target neonatal trough levels extrapolated from adult data $[3,10,11]$.

Risks associated with suboptimal trough levels involve treatment failure and emergence of resistant bacterial strains [7]. Based on the 2011 Infectious Diseases Society of America pediatric recommendations for trough targets of $10-20 \mathrm{mg} / \mathrm{mL}$ [11], and our clinical observations that recommended trough levels were not being achieved, our group conducted a study to assess the effectiveness of our 
Table 1 Previous empirical dosing regimen

\begin{tabular}{lll}
\hline $\begin{array}{l}\text { Chronological age, } \\
\text { days }\end{array}$ & Weight, g & $\begin{array}{l}\text { Dosing interval (with } 15 \mathrm{mg} / \mathrm{kg} \\
\text { dose) }\end{array}$ \\
\hline $0-7$ & $<1200$ & $24 \mathrm{~h}$ \\
& $1200-2000$ & $18 \mathrm{~h}$ \\
& $>2000$ & $12 \mathrm{~h}$ \\
$>7$ & $<1200$ & $24 \mathrm{~h}$ \\
& $1200-2000$ & $12 \mathrm{~h}$ \\
& $>2000$ & $8 \mathrm{~h}$
\end{tabular}

Reproduced and adapted from Dersch-Mills et al. [5]. Assessment of initial vancomycin dosing in neonates. Paeditr Child Heal. 2014;19(6):e30-4.

previous empirical dosing regimen $(15-45 \mathrm{mg} / \mathrm{kg} / \mathrm{day}$; Table 1) at achieving target levels [5]. The results indicated that $65 \%$ of neonates were not meeting the minimum trough recommendation upon initial dosing. Based on this, in 2014 our group developed a revised empirical dosage regimen $(20-60 \mathrm{mg} / \mathrm{kg} /$ day $)$ that was predicted to better achieve target trough levels (Table 2).

Other centers have attempted to assess the performance of empirical dosing regimens similar to ours [10, 12, 13]. A group in the Netherlands investigated a slightly different regimen (15 to $80 \mathrm{mg} / \mathrm{kg} / \mathrm{day})$, and found that $47 \%$ ( $n=$ 112) of neonates did not achieve target serum trough concentrations $(10-15 \mathrm{mg} / \mathrm{L})$ on their initial dose [8].

Clinical factors impacting renal function and vancomycin clearance should be considered when revising a dosing regimen. Gestational age (GA) and chronological age (CA) are known to be correlated with vancomycin clearance [4]. Neonates who are small for gestational age (SGA; birth weight $<10$ th percentile for GA) [6] have been shown to have impaired renal function [14]. Medications with nephrotoxic effects can also reduce renal function [15]. In addition, conventional mechanical ventilation has been shown to cause an increase in thoracic pressures, reducing cardiac output to the kidneys [16].

The initiative to improve our neonatal dosing practices began with our original study [5] which determined that we were not consistently reaching recommended target troughs. We implemented a new vancomycin regimen across all our sites, based on our own results and guiding standards from the literature, such as the Neofax recommendations [17]. We used data from a cohort of preterm and term babies with the primary objective of validating the effectiveness of our revised empirical regimen in achieving target trough levels.

\section{Methods}

\section{Study design}

A multisite retrospective before-and-after cohort study was conducted using chart review from January 2014 to January
2016 at five neonatal intensive care units (NICUs). Ethics approval was received from the local Health Research Ethics Board.

\section{Inclusion and exclusion criteria}

The study population consisted of neonates admitted to a NICU, up to a postmenstrual age (PMA) of 44 weeks. Patients on their initial course of vancomycin therapy who received a minimum of two doses in accordance with the revised vancomycin dosing regimen $(20$ to $60 \mathrm{mg} / \mathrm{kg} / \mathrm{day}$, Table 2) and had at least one appropriately drawn vancomycin level (drawn within $1 \mathrm{~h}$ prior to the pre-third or later dose) were included in the study. In cases where more than one vancomycin level was drawn, the first steady-state vancomycin level which was drawn appropriately was included. If this level was not within target, then the first steady-state vancomycin level that did reach target trough range was recorded for analysis of the dose adjustment that was required to reach a target trough level. Repeat courses of vancomycin were excluded.

\section{Data collection}

A list of patients who received vancomycin while in an NICU from January 2014 through January 2016 was generated through a computerized charting database. Demographic data collected included date of birth, GA, $\mathrm{CA}$, birth weight and weight at the time of vancomycin initiation. Data on clinical factors were collected and included: serum creatinine $(\mathrm{SrCr})$, concomitant use of nephrotoxic medications, SGA status, diagnosis of necrotizing enterocolitis (NEC), respiratory support and microbial cultures.

\section{Outcome measures}

\section{Primary outcome}

The primary outcome was to determine the percentage of neonates who achieved serum trough levels below target $(<10 \mathrm{mg} / \mathrm{L})$, at target $(10-20 \mathrm{mg} / \mathrm{L})$ or above target $(>20$ $\mathrm{mg} / \mathrm{L}$ ) on their initial vancomycin dose using our revised dosing regimen.

\section{Secondary outcomes}

1. Mean percent difference between the revised empiric dosing and the dose required to achieve target levels (if they were achieved).

2. Difference in mean trough levels between the two cohorts. 
Table 2 Revised dosing regimen used in the present cohort (created in 2014), mean troughs per age group and proportion of patients in the present cohort with trough levels below, at or above target

\begin{tabular}{|c|c|c|c|c|c|c|c|c|}
\hline Age group & PMA (weeks) & CA (days) & Dose (IV) and Interval & N 118 & Mean trough $(\mathrm{SD})$ & Level $<10$ & Level 10-20 & Level $>20$ \\
\hline 1 & $\leq 29$ & $0-21$ & $15 \mathrm{mg} / \mathrm{kg}$ dose every $18 \mathrm{~h}$ & 31 & $9.28(3.74)$ & $61.29 \%$ & $38.71 \%$ & $0.00 \%$ \\
\hline 2 & $\leq 29$ & $>21$ & $15 \mathrm{mg} / \mathrm{kg}$ dose every $12 \mathrm{~h}$ & 4 & $3.00(2.81)$ & $50.00 \%$ & $50.00 \%$ & $0.00 \%$ \\
\hline 3 & $30-36$ & $0-14$ & $15 \mathrm{mg} / \mathrm{kg}$ dose every $12 \mathrm{~h}$ & 14 & $12.56(4.13)$ & $21.43 \%$ & $78.57 \%$ & $0.00 \%$ \\
\hline 4 & $30-36$ & $>14$ & $15 \mathrm{mg} / \mathrm{kg}$ dose every $8 \mathrm{~h}$ & 29 & $29(17.25)$ & $10.34 \%$ & $68.97 \%$ & $20.69 \%$ \\
\hline 5 & $37-44$ & $0-7$ & $15 \mathrm{mg} / \mathrm{kg}$ dose every $12 \mathrm{~h}$ & 12 & $12(11.02)$ & $50.00 \%$ & $41.67 \%$ & $8.33 \%$ \\
\hline 6 & $37-44$ & $>7$ & $15 \mathrm{mg} / \mathrm{kg}$ dose every $8 \mathrm{~h}$ & 28 & $28(12.84)$ & $39.29 \%$ & $46.43 \%$ & $14.29 \%$ \\
\hline 7 & $\geq 45$ & ALL & $15 \mathrm{mg} / \mathrm{kg}$ dose every $6 \mathrm{~h}$ & 0 & NA & NA & NA & NA \\
\hline
\end{tabular}

$P M A$ postmenstrual age, $C A$ chronological age

3. Differences in proportion of patients achieving target trough levels on the previous empiric dosing regimen (historical cohort) compared to the revised (present cohort) vancomycin regimen.

4. Any relationships between demographic and clinical characteristics and trough levels in the present cohort, including:

- SGA

- Presence of nephrotoxic medications (diuretics, aminoglycosides, inotropes, steroids, non-steroidal anti-inflammatory agents) administered $<48 \mathrm{~h}$ prior to the included vancomycin trough.

- Highest level of respiratory support (invasive, noninvasive or spontaneous) administered $<48 \mathrm{~h}$ prior to the included vancomycin trough. Our definition of invasive respiratory support included assist control, pressure control, high-frequency oscillation, highfrequency jet ventilation and synchronized intermittent mandatory ventilation. Non-invasive respiratory support was defined as continuous positive airway pressure, bi-level ventilation and noninvasive positive pressure ventilator support.

- $\mathrm{SrCr}$ values within $48 \mathrm{~h}$ before or $24 \mathrm{~h}$ after the included vancomycin trough.

- Presence of positive blood, urine, internal wound or skin, cerebral spinal fluid, sputum and bronchoalveolar lavage cultures $(<48 \mathrm{~h}$ prior to initiation or during vancomycin therapy).

- Diagnosis of NEC $(<48 \mathrm{~h}$ prior to initiation or during vancomycin therapy).

- CA

- PMA

\section{Data analysis}

Statistical analyses were conducted using Microsoft Excel and SPSS Inc. (Chicago, Illinois). Descriptive statistics were used for patient characteristics and clinical descriptors. Baseline characteristics were compared between cohorts using $t$-test analysis (GA at birth, PMA, CA) and weight at the time of vancomycin initiation).

The primary endpoint was calculated for the cohort overall and for each GA/CA subgroup in our dosing regimen. For our secondary endpoints, mean percentage increase in total daily dose required to achieve target levels (if they were achieved) was calculated. Chi-squared and pairwise $z$-test post hoc analysis with Bonferroni correction was conducted to test for cohort differences in proportions and $t$-tests were used for cohort differences in means.

Finally, the relationship between demographic and clinical covariates with vancomycin trough levels was assessed using multiple linear regression. Variables associated with vancomycin troughs at $p<0.05$ in the simple linear regression were included in the multiple linear regression model for prediction of vancomycin troughs.

\section{Results}

A total of 194 patients were screened for inclusion, and 76 (39.16\%) participants were excluded. Twenty-nine (15\%) neonates were not started on the revised dosing regimen, $17(9 \%)$ did not have any trough levels collected, 27 (14\%) had inappropriately sampled trough levels and 3 (1.6\%) had drug levels collected prematurely. A total 118 neonates were included in the current cohort, with a median gestation age of 28.26 (Table 3). The present and historical cohorts differed in PMA, CA and weight at initiation of vancomycin but did not differ in GA at birth. The new dosing regimen was made up of seven age groups (Table 2); however, no patients in the present cohort met the age criteria of age group seven. Twentythree patients had positive cultures. Most bacterial isolates were Gram positive (74\%), with the most common being CoNS (39\% of isolates). Other Gram-positive isolates 
Table 3 Baseline characteristics

\begin{tabular}{llll}
\hline Patient characteristics & Present cohort $(N=118)$ & Historical cohort $(N=158)$ & $p$ value \\
\hline Gestational age at birth (weeks); median (IQR) & $28.4(26.3-34.3)$ & $28(26.0-31.9)$ & $31.1(27.6-35.1)$ \\
PMA at vancomycin initiation (weeks); median (IQR) & $33.4(29.1-38.5)$ & $12(9.0-21.0)$ & $p=0.114$ \\
CA at vancomycin initiation (days); median (IQR) & $15(8.0-37.5)$ & $1452.32(846.81)$ & $p=0.002$ \\
Weight at initiation of vancomycin (g); mean (SD) & $1814.20(961.43)$ & $p=0.001$ \\
\hline
\end{tabular}

$P M A$ postmenstrual age, $C A$ chronological age, $\mathrm{SrCr}$ serum creatinine, $I Q R$ interquartile range

Table 4 Cohort differences in mean troughs and proportion of patients reaching below, at and above target trough levels

\begin{tabular}{llll}
\hline & Present cohort & Historical cohort & $p$ value $^{\mathrm{a}}$ \\
\hline Below target (\%) & 37.29 & 64.50 & $p<0.05$ \\
At Target (\%) & 53.39 & 34.17 & $p<0.05$ \\
Above target (\%) & 9.32 & 1.30 & $p<0.05$ \\
Troughs; mean (SD) & $12.68(6.13)$ & $8.57(4.53)$ & $p=0.001$ \\
\hline
\end{tabular}

${ }^{a}$ Pairwise $z$-test comparisons with Bonferroni correction for proportion differences and Welch's $T$-test for trough differences

included MRSA, methicillin-sensitive Staphylococcus aureus and Enterococcus faecalis. Six patients had cultures showing Gram-negative isolates, including Escherichia coli, Enterobacter aerogenes and Klebsiella oxytoca.

In the present cohort, $63(53.39 \%)$ participants achieved target trough levels $(10-20 \mathrm{mg} / \mathrm{L}), 44(37.29 \%)$ reached levels of $<10 \mathrm{mg} / \mathrm{L}$ and $11(9.32 \%)$ had levels of $\geq 20 \mathrm{mg} / \mathrm{L}$ (Table 4). The youngest age group had the greatest proportion of patients $(61.29 \%)$ with trough levels of $<10 \mathrm{mg} / \mathrm{L}$ (Table 2). However, out of the 31 patients in this age group, only 3 had trough levels of $<5 \mathrm{mg} / \mathrm{L}$. On the opposite end, trough levels of $>20 \mathrm{mg} / \mathrm{L}$ were found in the highest proportion $(20.69 \%)$ in age group four, with five patients achieving troughs in the range of $20-26 \mathrm{mg} / \mathrm{L}$ and one patient with a trough of $45.7 \mathrm{mg} / \mathrm{L}$. Age group three was the most successful, with the greatest proportion $(78.57 \%)$ of patients reaching target trough levels.

Out of the 55 neonates who did not reach target troughs on the revised dosing regimen, 28 patients received a dose change to achieve target levels. The percent dose adjustment ranged from -29.63 to $71.43 \%$, with a mean of $27.28 \%$. Patients in the youngest age group required the greatest mean daily dose increase $(n=9 ; 50.40 \%)$, while patients in age group four required a mean dose decrease $(n=4 ;-26.61 \%)$.

Mean trough levels were compared between cohorts. A significant difference in mean troughs was found with higher levels seen in the present cohort ( $p<0.001$; Table 4$)$. In addition, chi-squared analysis demonstrated an association between trough levels and cohort group $(p<0.001)$, and post hoc pairwise comparisons demonstrated that the
Table 5 Associations between clinical/demographic covariates and trough levels

\begin{tabular}{|c|c|c|}
\hline Covariates $(n)$ & $\begin{array}{l}\text { Simple } \\
\text { regression }\end{array}$ & $\begin{array}{l}\text { Multiple } \\
\text { regression }\end{array}$ \\
\hline & $p$ value & $p$ value \\
\hline PMA (118) & 0.206 & \\
\hline CA (118) & 0.214 & \\
\hline $\begin{array}{l}\text { Weight at vancomycin initiation } \\
\text { (118) }\end{array}$ & 0.510 & \\
\hline Birth weight (118) & 0.753 & \\
\hline SGA (15) & 0.691 & \\
\hline Treatment for NEC (29) & 0.325 & \\
\hline $\begin{array}{l}\text { Serum creatinine } 48 \mathrm{~h} \text { prior to or } \\
24 \mathrm{~h} \text { post trough level ( } 44)\end{array}$ & 0.187 & \\
\hline Nephrotoxic medications (59) & 0.346 & \\
\hline $\begin{array}{l}\text { Spontaneous respiratory support } \\
\text { (18) }\end{array}$ & 0.315 & \\
\hline $\begin{array}{l}\text { Non-invasive respiratory support } \\
\text { (15) }\end{array}$ & 0.186 & \\
\hline Invasive respiratory support (68) & $0.033 *$ & 0.139 \\
\hline Bacterial culture status (20) & $0.025 *$ & $0.031 *$ \\
\hline
\end{tabular}

$P M A$ postmenstrual age, $C A$ chronological age, $S G A$ small for gestational age, $N E C$ necrotizing enterocolitis

*Indicates significant associations $(p<0.05)$

proportions of patients reaching below, at and above target troughs differed significantly between cohorts $(p<0.05$ for all comparisons). The present cohort had a higher proportion of troughs at and above target, and a lower proportion of below target troughs in comparison to the historical cohort.

Simple regression identified culture status and invasive respiratory support as the two covariates significantly associated with vancomycin troughs $(p<0.05)$, and thus these were selected for inclusion in the multiple regression analysis. Culture status remained the only statistically significant predictor of vancomycin troughs $(p=0.031)$ in the multiple regression analysis. Culture status was negatively associated with vancomycin levels $(\beta=-3.271 ; p=$ 0.031 ), indicating that patients with positive cultures were more likely to have lower trough levels than patients with negative cultures (Table 5). 


\section{Discussion}

In 2009, we set out to confirm and address a care gap observed in our neonatal population. The original pharmacokinetically predicted regimen which was suggested in our previous study [5] differs slightly from the current regimen as the current regimen was informed by the greater body of evidence to attempt alignment of the various dosing regimens in the literature. The current analysis identified a substantial improvement in the proportion of neonates who achieved target vancomycin levels on the current regimen compared to the previous ( $53.39 \%$ vs $34.17 \%$ respectively). The proportion of patients reaching levels of $<10 \mathrm{mg} / \mathrm{L}$ was much lower with the new regimen $(37.29 \%$ vs $64.50 \%)$, and the proportion of patients with troughs $>20 \mathrm{mg} / \mathrm{L}$ did not surpass $10 \%$.

Our dosing regimen closely resembles the Neofax ${ }^{\circ}$ vancomycin dosing recommendations, with differences in the CA cut-off points for age groups one and two [17]. Other studies have based dosing regimens on the Neofax protocol $[10,12,13]$. In one study, the dosing regimen was similar to ours with the exception of a lower standard dose $(10 \mathrm{mg} / \mathrm{kg}$ vs $15 \mathrm{mg} / \mathrm{kg})$, which may explain why the majority of their participants $(76 \%)$ achieved troughs of $<10 \mathrm{mg} / \mathrm{L}$ [10]. Ringenberg et al. [12] evaluated the effectiveness of the Neofax dosing model using $15 \mathrm{mg} / \mathrm{kg}$ as the standard empiric dose. However, even with this dosing increase, $71.9 \%(n=171)$ of patients achieved troughs values of $<10 \mathrm{mg} / \mathrm{L}$ after empiric dosing [12]. The dose required to achieve initial target troughs was found to be an average dose of $32.4 \mathrm{mg} / \mathrm{kg} /$ day. Similarly, Badran et al. [13] found that $16.6 \%$ of 151 neonates achieved target troughs between 10 and $16.6 \mathrm{mg} / \mathrm{L}$ using an empiric dose based on Neofax ${ }^{\circ}$.

Although our current regimen achieved better results in comparison to the previous regimen, a proportion of patients still achieved sub-target troughs. The patients with the greatest proportion of troughs of $<10 \mathrm{mg} / \mathrm{L}$ were those in age group one (Table 2). A pharmacodynamics study using 1000 simulated neonates and C-reactive protein as an endpoint for vancomycin efficacy demonstrated that neonates $<29$ weeks PMA receiving $15 \mathrm{mg} / \mathrm{kg}$ every $24 \mathrm{~h}$ are generally underdosed [18]. The authors suggested optimizing the dosing regimen to $15 \mathrm{mg} / \mathrm{kg}$ every $12 \mathrm{~h}$ for neonates < 29 weeks. Comparably, another study suggested $12.5 \mathrm{mg} / \mathrm{kg}$ every $12 \mathrm{~h}$ for all neonates with PMA <29 weeks [19]. Based on this, it may be beneficial to further revise our dosing regimen to include shorter dosing intervals for patients in age group one. In the Neofax ${ }^{\circ}$ dosing regimen, patients $<29$ weeks are subdivided into CA groups of $<14$ and $>14$ days [17]; however, in our regimen this group is subdivided as $<21$ and $>21$ days. Perhaps patients who are $<29$ weeks
(PMA) and >14 days (CA) experience changes in renal function which renders them underdosed with our current regimen.

Having said that, it is important to examine the evidence behind the targets we aim to achieve. Evidence has emerged supporting lower target troughs of 7-11 $\mathrm{mg} / \mathrm{L}$ for achievement of AUC/MIC $>400$ in neonates when treating MRSA infections with an MIC $\leq 1$ [9]. Further research is needed in this area to determine if clinical outcomes are comparable with lower target troughs in neonates.

Finally, we assessed the relationship between clinical characteristics and vancomycin troughs. Microbial culture status was the only statistically significant covariate in our multiple regression model, with positive cultures showing an association with lower vancomycin troughs. This is consistent with the theory that sepsis may increase volume of distribution [20].

Due to the retrospective nature of the study, potential confounders affecting vancomycin trough levels could not be controlled for. The two cohorts differed in PMA, CA and weight, but these factors did not have a significant association with trough levels in the simple linear regression analysis. Many vancomycin troughs were collected prior to administration of the third dose which may have underestimated levels at true steady state. Thus, the proportion of babies achieving target (or supra-therapeutic) levels when using the revised regimen may be greater than is shown in our results. This is, however, the usual level (pre-third) used by our group and reflects current practice. Furthermore, time to steady state can vary significantly in neonates, depending on postnatal age; thus true steady-state levels would require individualized timing of level collection [21]. In addition, our regression analyses may not have been powered to detect every significant covariate.

Lastly, although $\mathrm{SrCr}$ was analyzed for association with serum trough levels, only 44 patients (37\%) had $\mathrm{SrCr}$ samples collected within $48 \mathrm{~h}$ before and $24 \mathrm{~h}$ after the included vancomycin trough. Vancomycin troughs and $\mathrm{SrCr}$ were not statistically associated, and thus based on our results we cannot indicate if supra-therapeutic levels led to potential nephrotoxicity in the present cohort. It is possible that an association was not seen due to a low sample of patients with collected $\mathrm{SrCr}$.

Our empiric vancomycin dosing regimen is significantly more effective in achieving serum trough concentrations than the previous one. Further adjustments could be made to increase the proportion of patients reaching target troughs. We have created a regimen that balances timely achievement of target troughs for a large proportion of our population while remaining close to published vancomycin dosing regimens. We hope this will inform other centers and allow the convergence of various dosing regimens in this unique population. 
Funding This study was supported by the Pharmacy Services at Alberta Health Services.

Author contributions LR participated in the initial study design, conducted data collection and statistical analysis, drafted the initial manuscript and reviewed and revised the manuscript. DD-M participated in initial project design, oversaw data collection and statistical analysis, reviewed and revised the manuscript. TB contributed to initial project design, reviewed and revised the manuscript. AA and KY contributed to initial project design, and reviewed the manuscript. BA contributed to initial project design, assisted with statistical analysis and reviewed the manuscript. All authors approved the final manuscript as submitted and agree to be accountable for all aspects of the work.

\section{Compliance with ethical standards}

Conflict of interest The authors declare that they have no conflict of interest.

\section{References}

1. Bizzarro MJ, Raskind C, Baltimore RS, Gallagher PG. Seventyfive years of neonatal sepsis at Yale: 1928-2003. Pediatrics. 2005;116:595-602.

2. Bizzarro MJ, Shabanova V, Baltimore RS, Dembry LM, Ehrenkranz RA, Gallagher PG. Neonatal sepsis 2004-2013: the rise and fall of coagulase-negative staphylococci. J Pediatr. 2015;166:1193-9.

3. Jacqz-Aigrain E, Zhao W, Sharland M, van den Anker JN. Use of antibacterial agents in the neonate: 50 years of experience with vancomycin administration. Semin Fetal Neonatal Med. 2013;18:28-34.

4. de Hoog M, Mouton JW, van den Anker JN. Vancomycin: pharmacokinetics and administration regimens in neonates. Clin Pharmacokinet. 2004;43:417-40.

5. Dersch-Mills D, Bengry T, Akierman A, Alshaikh A, Yusuf K. Assessment of initial vancomycin dosing in neonates. Paeditr Child Heal. 2014;19:e30-4.

6. Kim J, Walker SA, Iaboni DC, Walker SE, Elligsen M, Dunn MS, et al. Determination of vancomycin pharmacokinetics in neonates to develop practical initial dosing recommendations. Antimicrob Agents Chemother. 2014;58:2830-40.

7. Rybak M, Lomaestro B, Rotschafer JC, Moellering R, Craig W, Billeter M, et al. Therapeutic monitoring of vancomycin in adult patients: a consensus review of the American Society of HealthSystem Pharmacists, the Infectious Diseases Society of America, and the Society of Infectious Diseases Pharmacists. Am J Heal Pharm. 2009;66:82-98.
8. Sinkeler FS, de Haan TR, Hodiamont CJ, Bijleveld YA, Pajkrt D, Mathôt RAA. Inadequate vancomycin therapy in term and preterm neonates: a retrospective analysis of trough serum concentrations in relation to minimal inhibitory concentrations. BMC Pediatr. 2014; $14: 193$

9. Frymoyer A, Hersh AL, El-Komy MH, Gaskari S, Su F, Drover $\mathrm{DR}$, et al. Association between vancomycin trough concentration and AUC in neonates. Antimicrob Agents Chemother. 2014;58:6454-61.

10. Vandendriessche A, Allegaert K, Cossey V, Naulaers G, Saegeman V, Smits A. Prospective validation of neonatal vancomycin dosing regimens is urgently needed. Curr Ther Res Clin Exp. 2014;76:51-7.

11. Liu C, Bayer A, Cosgrove SE, Daum RS, Fridkin SK, Gorwitz RJ, et al. Clinical practice guidelines by the infectious diseases Society of America for the treatment of methicillin-resistant Staphylococcus aureus infections in adults and children. Clin Infect Dis. 2011;52:e18-55.

12. Ringenberg T, Robinson C, Meyers R, Degnan L, Shah P, Siu A, et al. Achievement of therapeutic vancomycin trough serum concentrations with empiric dosing in neonatal intensive care unit patients. Pediatr Infect Dis J. 2015;34:742-7.

13. Badran EF, Shamayleh A, Irshaid YM. Pharmacokinetics of vancomycin in neonates admitted to the neonatology unit at the Jordan University Hospital. Int $\mathbf{J}$ Clin Pharmacol Ther. 2011;49:252-7.

14. Aly H, Davies J, El-Dib M, Massaro A. Renal function is impaired in small for gestational age premature infants. J Matern Fetal Neonatal Med. 2013;26:388-91.

15. Safina AI, Daminova MA, Abdullina GA. Acute kidney injury in neonatal intensive care: medicines involved. Int J Risk Saf Med. 2015;27 Suppl 1:S9-10.

16. Koyner JL, Murray PT. Mechanical ventilation and lung-kidney interactions. Clin J Am Soc Nephrol. 2008;3:562-70.

17. Young TE, Magnum R, eds. Neofax, 24th Edition. Montvale: Thomson Reuters; 2011.

18. Ramos-Martin V, Johnson A, Livermore J, McEntee L, Goodwin $\mathrm{J}$, Whalley S, et al. Pharmacodynamics of vancomycin for CoNS infection: experimental basis for optimal use of vancomycin in neonates. J Antimicrob Chemother. 2016;71:992-1002.

19. Schwartz ML, Wrobel J, Huntley J, Zeilmann C. Protocol versus nonprotocol dosing of vancomycin in neonates: a single center evaluation of steady state trough levels. Am J Perinatol. 2015;33:678-82.

20. Roberts JA, Lipman J. Antibacterial dosing in intensive care: pharmacokinetics, degree of disease and pharmacodynamics of sepsis. Clin Pharmacokinet. 2006;45:755-73.

21. Janssen EJ, Välitalo PA, Allegaert K, De Cock RF, Simons SH, Sherwin CM, et al. Towards rational dosing algorithms for vancomycin in neonates and infants based on population pharmacokinetic modeling. Antimicrob Agents Chemother. 2016;60:1013-21. 